\title{
Polarizing neutron monochromator with reduced higher-order contributions
}

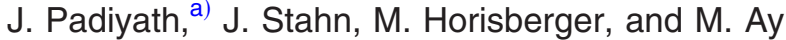 \\ Laboratory for Neutron Scattering, ETHZ \& PSI, 5232 Villigen, Switzerland \\ P. Böni \\ Physics Department E21, TUM, James-Frank Str., 85748 Garching, Germany
}

(Received 4 September 2006; accepted 17 November 2006; published online 20 December 2006)

\begin{abstract}
Polarized neutron reflectivity experiments have been performed to investigate polarizing multilayer monochromators. The neutron monochromators have been sputtered with $\mathrm{Ti}$ and $\mathrm{Fe}-\mathrm{Co}-\mathrm{V}$ as layer materials with a nominal period of $11 \mathrm{~nm}$. For more than two layers per period, the bulk magnetization of $2.35 \mu_{B}$ is reduced to $1.6 \mu_{B}$. With 22 layers per period and 15 periods the higher-order contributions are decreased to below $0.6 \%$ of the first-order Bragg peak intensity (reflectivity of 0.21 ) at $q_{z}=0.06 \AA^{-1}$. The polarization efficiency for the final monochromator is 95.5\%. (C) 2006 American Institute of Physics. [DOI: 10.1063/1.2410235]
\end{abstract}

In neutron scattering facilities, neutron reflectometer usually has a monochromator and a Be filter to select a certain wavelength. ${ }^{1-3}$ Especially for the experiments related to neutron reflectometry, monochromators based on multilayers play an important rôle. ${ }^{4-8}$ One of the major problems in realizing a truely monochromatic beam are the higher-order reflections from multilayers as well as from single crystals. In the present case a unique approach to suppress higherorder reflections is given. By dividing the bilayer period of a multilayer monochromator into several layers with individual thicknesses, a quasisinusoidal scattering length density profile can be obtained leading to a fundamental monochromator (only the first-order Bragg peak has significant intensity). ${ }^{9,10}$ This is valid for one spin state where the firstorder Bragg peak has a high reflectivity due to the scattering potential difference from magnetized $\mathrm{Fe}-\mathrm{Co}-\mathrm{V}$ to Ti. For the other spin state the scattering potential difference between the two layers is almost zero. The multilayer were deposited on Si (100) wafers using Ar ion dc magnetron sputtering and the layer materials are $\mathrm{Fe}-\mathrm{Co}-\mathrm{V}$ and $\mathrm{Ti}$.

In order to ensure the homogeneity of the individual layers, the substrates were mounted on a moving table underneath the sputtering target. Small slits (width set to $12 \mathrm{~mm}$ ) were placed in front of the targets to reduce the sputtering rate, allowing to deposit very thin layers down to $2 \AA$. The deposition was carried out with Ar gas at 50 SCCM (SCCM denotes cubic centimeter per minute at STP) (pressure within the chamber $\approx 3 \times 10^{-3}$ mbar). A series of six multilayers has been prepared with a different number of layers per period $(2,6,10,14,18$, and 22) but the same nominal period.

To verify the validity of the approach, specular and offspecular polarized neutron reflectivity measurements have been performed on the neutron reflectometer ADAM (fixed wavelength of $4.4 \AA$ ) at the ILL in France and on AMOR (time-of-flight mode ${ }^{11}$ ) at SINQ in Switzerland. ${ }^{12}$ Polarized neutron reflectivity is ideally suited to investigate the magnetic structure of grown superlattices. ${ }^{13-15}$

The polarized neutron reflectivity measurements gave information about the magnetic moments and structures within the buried layers. The scattering length densities from the fit of the specular reflectivity of a stacked bilayer are nuclear

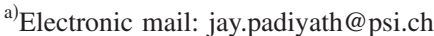

$4.65 \times 10^{-6} \AA^{-2}$ and magnetic $4.45 \times 10^{-6} \AA^{-2}$ for $\mathrm{Fe}-\mathrm{Co}-\mathrm{V}$, the magnetic scattering length of the interface layer is set to zero (magnetic dead layer), and the scattering length density of $\mathrm{Ti}$ is found to be $-1.9 \times 10^{-6} \AA^{-2}$. The magnetic scattering length density of the $\mathrm{Fe}-\mathrm{Co}-\mathrm{V}$ corresponds to $1.6 \mu_{B}$. Investigations by Rücker et al. ${ }^{16}$ gave $4.1 \times 10^{-6} \AA^{-2}$ for the magnetic scattering length density of $\mathrm{Fe}-\mathrm{Co}-\mathrm{V}$, which is significantly smaller than the expected nominal value for $\mathrm{Fe}-\mathrm{Co}-\mathrm{V}$ of $5.6 \times 10^{-6} \AA^{-2}$.

The off-specular results are shown in Fig. 1. Top figures are results from a stacked bilayer. (a) is the intensity map of the spin down state. The intensities are smaller by a factor of 10 compared to the spin up state presented in Fig. 1(b). In the latter, the Yoneda wings and Bragg sheets are observed, indicating uncorrelated and correlated roughnesses. The higher-order contributions are seen in both figures as dots at $q_{z}=0.095 \AA^{-1}$ (second order) and $q_{z}=0.14 \AA^{-1}$ (third order).

Below, the off-specular intensity maps of the fundamental polarizing monochromator (22 layers per period) are shown for both spin states [spin down (c) and spin up (d)]. The first-order Bragg peak is at $q_{z}=0.06 \AA^{-1}$ (reflectivity of 0.21 ) and the polarization efficiency of the multilayer is $95.5 \%$. The second order, at $q_{z}=0.12 \AA^{-1}$, has $0.6 \%$ of the first-order Bragg peak intensity (spin up state). This peak is not of magnetic origin but rather due to magnetic dead layers at the interfaces. The third-order Bragg peak is expected at $q_{z}=0.18 \AA^{-1}$, but no intensity is observed. The Bragg sheet, indicating correlated roughness, has less than $0.5 \%$ intensity compared to the first-order Bragg peak at $q_{z}=0.06 \AA^{-1}$.

For the fundamental monochromator, the total thickness of the magnetic layers is reduced from 55 to $26 \AA$ per period (obtained from simulations, magnetic dead layer of $29 \AA$ ). If this value is taken for the magnetization experiments to evaluate the magnetization of the $\mathrm{Fe}-\mathrm{Co}-\mathrm{V}$, the result is 1.6(1) $\mu_{B}$. This corresponds to a magnetic scattering length density of $4.4(2) \times 10^{-6} \AA^{-2}$, which is the value used for simulations and is identical within the errors to the magnetization in the bilayer case. A mixing of the $\mathrm{Fe}-\mathrm{Co}-\mathrm{V}$ layers with the Ti leads to the suppression of the magnetization as observed by Stahn and Clemens. ${ }^{17}$

The magnetization of the as-deposited multilayers has been measured by bulk magnetization measurements. For the case with two layers per period, 1.45(1) $\mu_{B}$ is observed, while 

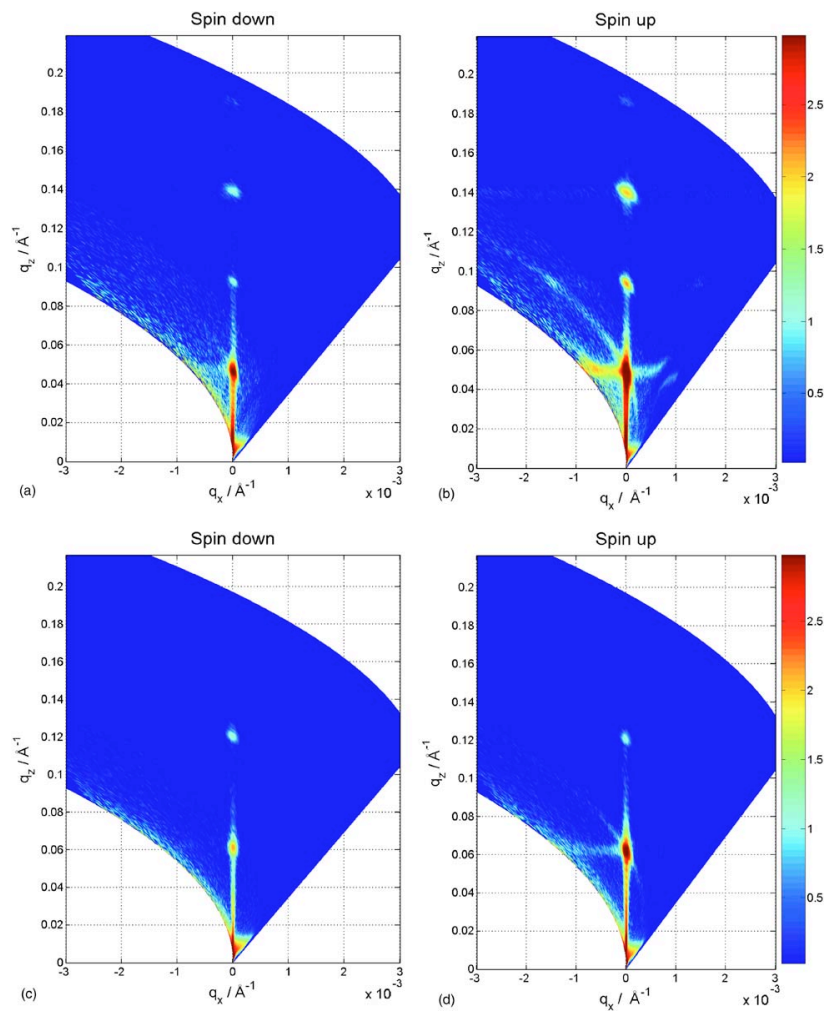

FIG. 1. (Color online) Off-specular plots of the multibilayer with the same intensity scale. (a) is the intensity map of the spin down state. (b) is the intensity map of the spin up state. For both states, the higher-order contributions are observed. The difference in intensity of the two spin states is a factor of 10. In (b), the Bragg sheets and Yoneda wings are visible (roughness). (c) Off-specular reflectivity of the multilayer with 22 layers per period (15 periods) for the spin down reflectivity and (d) shows the spin up reflectivity. The second-order Bragg peak at $q_{z}=0.12 \AA^{-1}$ has the same intensity for both spin states. The third-order Bragg peak is expected at $q_{z}=0.18 \AA^{-1}$ but is completely invisible. The Bragg sheets in the spin up state has less than $0.5 \%$ of the intensity of the first Bragg peak.

in the cases with more than ten layers per period only $0.8(1) \mu_{B}$ is measured. For this evaluation the whole thickness of all $\mathrm{Fe}-\mathrm{Co}-\mathrm{V}$ layers for one period has been summed up. By taking the magnetic dead layers into account, the observed magnetization during the magnetization experiments is increased to above $1.5(2) \mu_{B}$. This is in agreement with results from the neutron experiments and with the multilayer experiments by Schanzer et al. ${ }^{18}$ They applied up to $700 \mathrm{Oe}$ and used $\mathrm{Fe}-\mathrm{Co}-\mathrm{V}$ layer thicknesses from 3 to $10 \mathrm{~nm}$ and observed reduced magnetic moments between $1.3 \mu_{B}$ and $1.9 \mu_{B}$ in a multilayer with a $5 \mathrm{~nm}$ period. Schanzer et al. suggested a multidomain state of the magnetic layers, in which the average component of the magnetization parallel to the applied field is reduced. These magnetic moments are reduced when compared to $\mathrm{Fe}-\mathrm{Co}-\mathrm{V}$ bulk measurements which give $2.35 \mu_{B}{ }^{19}$

For transmission electron microscopy (TEM) a Philips CM30ST with a voltage of $300 \mathrm{kV}$ has been used with a point-to-point resolution of $1.9 \AA$. The sample preparation for the measurements was performed by ion etching with $\mathrm{Ar}$ ions with a voltage of $4.3 \mathrm{kV}$. These studies have been performed to investigate the sputtered layer structures. TEM images of the $\mathrm{Fe}-\mathrm{Co}-\mathrm{V} / \mathrm{Ti}$ multilayer are presented in Fig. 2(a) is an image of a multibilayer, where the darker shades correspond to the $\mathrm{Fe}-\mathrm{Co}-\mathrm{V}$ layers. The sharp structures within the $\mathrm{Fe}-\mathrm{Co}-\mathrm{V}$ layers are small crystal grains
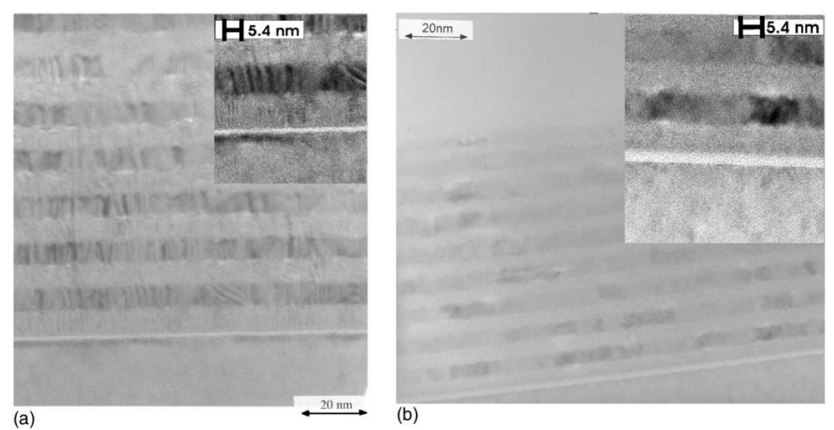

FIG. 2. (Color online) (a) High resolution transmission electron microscopy (HRTEM) image of a multibilayer. The darker layers are the $\mathrm{Fe}-\mathrm{Co}-\mathrm{V}$. The structures with stronger diffraction contrast indicate a crystallization of the $\mathrm{Fe}-\mathrm{Co}$. (b) HRTEM image of the fundamental monochromator. In this case, the crystallization is not as distinctive as in (a). The insets in both images are enlargements of the TEM images of the first four layers above the substrate. The interfaces are more blurred in (b).

within the layers. The Ti layers show a smaller degree of crystallization. Figure 2(b) shows a TEM image of the sample with 22 layers per period. The presented layers in the insets show the first sputtered layers above the substrate. The layers which are farther away from the substrate show less crystallization, caused by increasing roughness. Therefore the number of nucleation centers is increasing and the grains are constricting each other during the growth.

An electron diffraction (ED) image of the multibilayer is shown in Fig. 3(a). The circles indicate the peaks which are labeled within the image. The FeCo (110) direction and the Ti (100) intensities are clearly visible. An electron diffraction image of the fundamental monochromator is presented in Fig. 3(b). The crystallites are randomly oriented and, instead of spots, a ring is observed. No discrimination between the $\mathrm{Ti}$ and the $\mathrm{Fe}-\mathrm{Co}-\mathrm{V}$ can be made.

Interestingly, annealing lead to strongly enhanced growing of the crystallites as observed in the x-ray diffraction pattern. The results of the grain size calculation (by the Scherrer formula ${ }^{20,21}$ ) within the layers are presented in Table I. The full width at half maximum (FWHM) and the peak positions are listed. The grain growth is enhanced during the annealing process at $523 \mathrm{~K}$ from $1.4(1) \mathrm{nm}$ in the as-prepared state to an averaged grain size of $4.4(2) \mathrm{nm}$. These results lead to the conclusion that annealing cannot be
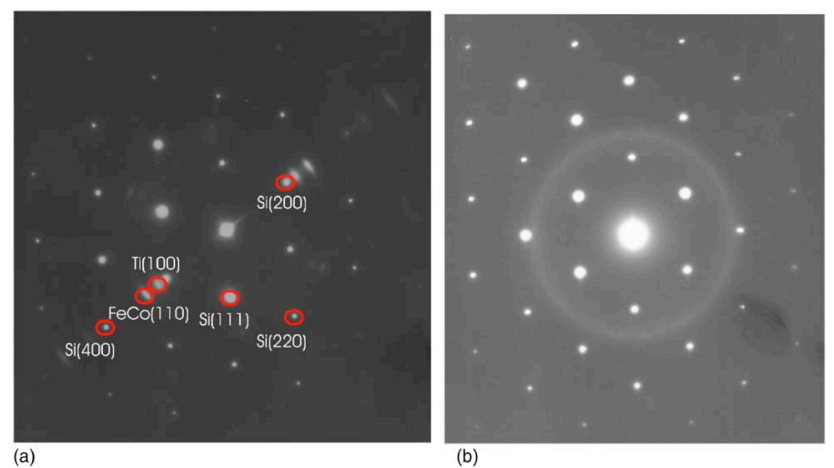

FIG. 3. (Color online) (a) Electron diffraction pattern of the multibilayer. Clearly visible are the FeCo (110) and the Ti (100) intensities. (b) Electron diffraction image of the fundamental multilayer. No discrimination between the $\mathrm{FeCo}$ and the $\mathrm{Ti}$ is possible. The crystallites are randomly oriented and instead of bright spots a diffuse ring is observed, which indicates that the crystallinity is very low. 
TABLE I. List of the peak positions and the calculated grain size for the multilayer monochromator with 22 layers per period in the as-prepared state and after the annealing process. The grain growth is enhanced by the annealing process.

\begin{tabular}{cccc}
\hline \hline Peak & Peak position $(\mathrm{deg})$ & FWHM $(\mathrm{deg})$ & Grain size $(\mathrm{nm})$ \\
\hline FeCo 110 (273 K) & $44.0(8)$ & $5.90(4)$ & $1.43(1)$ \\
FeCo 210 (273 K) & $77.6(9)$ & $7.34(4)$ & $1.37(7)$ \\
& & & \\
FeCo 110 (523 K) & $44.9(3)$ & $1.93(3)$ & $4.4(2)$ \\
FeCo 211 (523 K) & $82.6(9)$ & $1.93(7)$ & $5.4(7)$ \\
\hline \hline
\end{tabular}

used to blur the interfaces. It rather would lead to sharper interfaces and increased higher-order contribution due to the grain growth. This is explained by a phase separation at the interfaces.

In summary, the division of discrete bilayers into several layers in order to blur the magnetic scattering length at the interfaces in polarizing multilayers was applied in a periodic multilayer. TEM showed the blurring of the interfaces. ED measurements confirmed the random orientation of the very small $\mathrm{Fe}-\mathrm{Co}-\mathrm{V}$ grains $[1.4(1) \mathrm{nm}]$ in the case of the fundamental monochromator. Polarized neutron reflectivity experiments gave information about the magnetic structure within the buried layers. It showed that the reduced magnetization observed in the bulk magnetization experiments is due to the $\mathrm{Fe}$ and Co atoms close to the interface where the magnetization is strongly reduced (magnetic dead layers). This leads to the observed second-order Bragg peak for both spin states in the polarized reflectivity experiments. Therefore the approach is a significant step towards a fundamental polarizing monochromator. The second-order Bragg peak can probably be reduced by doping the interfaces with different magnetic materials which carry a higher magnetic moment to suppress the magnetic dead layers. Annealing of the structure cannot be used to blur the interfaces further, because phase separation at the interfaces leads to an increased grains size and sharper interfaces.

The authors thank M. Wolff for the help during the neutron experiments at the ILL and E. Müller for the TEM ex- periments. Financial support by the Swiss National Science Foundation is gratefully acknowledged (200021-101567). This work was supported by the European Union within the Sixth Framework Program FP6 under Contract No. 505925. This work was performed at the SINQ, Paul Scherrer Institute, Villigen, Switzerland and the ILL, Grenoble, France.

${ }^{1}$ L. D. Cussen, D. J. Goossens, and T. J. Hicks, Nucl. Instrum. Methods Phys. Res. A 440, 409 (2000).

${ }^{2}$ P. Mikula, J. Kulda, P. Lukas, M. Vrana, and V. Wagner, Nucl. Instrum. Methods Phys. Res. A 338, 18 (1994).

${ }^{3}$ H. Maier-Leibnitz, Ann. Acad. Sci. Fenn., Ser. A6 267, 1 (1967).

${ }^{4}$ I. Mirebeau, S. Itoh, S. Mitsuda, T. Watanabe, Y. Endoh, M. Hennion, and P. Calmettes, Phys. Rev. B 44, 5120 (1991).

${ }^{5}$ Neutrons in Biology, Basic Life Sciences Vol. 27, edited by B. P. Schoenborn (Plenum, New York, 1976), 125.

${ }^{6}$ D. Clemens, Physica B 221, 507 (1996).

${ }^{7}$ A. A. van Well, V. O. de Haan, H. Fredrikze, and D. Clemens, Physica B 283, 282 (2000).

${ }^{8}$ A. K. Freund, Nucl. Instrum. Methods 124, 93 (1975).

${ }^{9}$ J. Padiyath, J. Stahn, P. Allenspach, M. Horisberger, and P. Böni, Physica B 357, 218 (2005).

${ }^{10}$ J. Padiyath, J. Stahn, P. Allenspach, M. Horisberger, and P. Böni, Appl. Phys. Lett. 89, 113123 (2006).

${ }^{11}$ M. Gupta, T. Gutberlet, J. Stahn, P. Keller, and D. Clemens, Pramana, J. Phys. 63, 47 (2004).

${ }^{12}$ W. E. Fischer, Physica B 234-236, 1202 (1997).

${ }^{13}$ C. F. Majkrzak, J. Kwo, M. Hong, Y. Yafet, D. Gibbs, C. L. Chien, and J. Bohr, Adv. Phys. 40, 99 (1991).

${ }^{14}$ A. Schreyer, C. F. Majkrzak, Th. Zeidler, T. Schmitte, P. Bodeker, K. Theis-Brohl, A. Abromeit, J. A. Dura, and T. Watanabe, Phys. Rev. Lett. 79, 4914 (2002).

${ }^{15}$ K. V. O'Donovan, J. A. Borchers, C. F. Majkrzak, O. Hellwig, and E. F. Fullerton, Phys. Rev. Lett. 88, 067201 (2002).

${ }^{16}$ U. Rücker, E. Kentzinger, B. Toperverg, F. Ott, and T. Brückel, Appl. Phys. A: Mater. Sci. Process. 74, 607 (2002).

${ }^{17}$ J. Stahn and D. Clemens, Appl. Phys. A: Mater. Sci. Process. 74, 1532 (2002).

${ }^{18}$ C. Schanzer, V. R. Shah, P. Böni, and T. Krist, Physica B 350, E221 (2004).

${ }^{19}$ M. Senthil Kumar, V. R. Shah, C. Schanzer, P. Böni, T. Krist, and M. Horisnberger, Physica B 350, E241 (2004).

${ }^{20}$ B. D. Cullity, Elements of X-Ray Diffraction, 2nd ed. (Addison-Wesley, Reading, MA, 1978), 1, p. 102.

${ }^{21}$ B. E. Warren, X-ray Diffraction (Addison-Wesley, Reading, MA, 1969), p. 253. 